\title{
No impact of nasal septoplasty on the outcome of endoscopic dacryocystorhinostomy
}

\author{
Tal Koval ${ }^{1,2}$ - Ofira Zloto ${ }^{1,2} \cdot$ Arkadi Yakirevitch $^{2,3} \cdot$ Guy J. Ben Simon ${ }^{1,2} \cdot$ Joseph Ben-Shoshan $^{3} \cdot$ Elad Ben Artsi $^{1,2}$. \\ Alon Weissman ${ }^{1,2} \cdot$ Ayelet Priel $^{1,2}$
}

Received: 1 April 2019 / Revised: 29 July 2019 / Accepted: 14 October 2019 / Published online: 25 November 2019

(c) The Author(s), under exclusive licence to The Royal College of Ophthalmologists 2019

\begin{abstract}
Objective To compare the outcomes of combined endoscopic dacryocystorhinostomy (endoDCR) with nasal septoplasty for deviation of the nasal septum to endoDCR alone in cases of nasolacrimal duct obstruction (NLDO).

Methods A retrospective cohort study that included 107 consecutive patients with NLDO, who underwent endoDCR with or without concomitant nasal septoplasty in our institution between October 2009 and October 2017.

Results A total of 117 operations were performed (107 patients, 80.4\% females; mean age \pm SD $51.1 \pm 19.5$ years). Twentyfive (21.4\%) endoscopic surgeries were combined with septoplasty (the endoDCR + septoplasty group), and 92 (78.6\%) comprised endoDCR alone (the endoDCR group). There was no difference in anatomical success and functional success rates between the two groups ( $P=0.76$ and $P=0.18$, respectively). There were no complications attributed to the septoplasty component of the surgical procedure.

Conclusion Considerable numbers of patients undergoing endoDCR also require a septoplasty. Combining an additional procedure (septoplasty), that was not performed for its original indication but rather for facilitating the main surgical intervention (endoDCR), yields surgical success and associated complications equivalent to those of endoDCR alone.
\end{abstract}

\section{Introduction}

Improvements in endonasal visualization technology and in endonasal instrumentation have led to the increasing popularity of transnasal endoscopic dacryocystorhinostomy (endoDCR) procedures among oculoplastic surgeons. An endoscopic approach has the advantages of not requiring an external incision (as in the case for traditional external DCRs), preservation of the tear pump mechanism, and the ability to treat nasal comorbidities in order to improve the DCR outcome [1]. Concomitant deviation of the nasal septum (DNS) to the side of nasolacrimal duct obstruction (NLDO), however, may obscure visualization of the

Ayelet Priel

priel.ayelet@gmail.com

1 Goldschleger Eye Institute, Sheba Medical Center, Ramat Gan, Israel

2 Sackler Faculty of Medicine, Tel Aviv University, Tel-Aviv, Israel

3 Department of Otolaryngology \& Head and Neck Surgery, Sheba Medical Center, Ramat Gan, Israel insertion of middle turbinate (axilla) to the lateral nasal wall and hinder exposure of the lacrimal sac fossa, thereby jeopardizing surgical success by an inadequate rhinostomy $[2,3]$. Previous reports have suggested that anatomical failure of external and endoDCRs may be attributed to severe septal deviation, among other nasal structural abnormalities [4, 5]. Severe DNS was also significantly more frequent postoperatively in cases of previously failed external DCRs than in previously failed endoscopic DCRs [6]. The ability to perform a concomitant endoscopic septoplasty for correction of a DNS has been considered an additional advantage of endoDCR compared with the external approach by alleviating symptoms that may be related to DNS. More importantly, septoplasty can be performed to improve the execution and the outcome of the endoscopic DCR, even in patients lacking nasal obstruction symptoms $[2,3,5,7]$.

An endoDCR alone has never before been compared with an endoDCR combined with septoplasty in terms of surgical success and complication rates. Therefore, the primary goal of the current study was to carry out these analyses. Our assumption was that combining septoplasty with an endoDCR (when needed) in order to improve access 
and visualization would achieve success similar to endoDCR alone in the absence of a DNS, since the nasal structure would be similar in both groups after a septoplasty. We assumed that adding a septoplasty would reduce postoperative adhesion formation due to widening of the nasal passage and distancing the medial and lateral mucosal layers from each other.

\section{Materials and methods}

\section{Participants and study design}

A retrospective review was performed of the medical records of all consecutive patients with NLDO who underwent endoDCR at the Oculoplastic, Lacrimal, and Orbit Unit at the Goldschleger Eye Institute, Sheba Medical Center, between October 2009 and October 2017. A total of 117 operations were included. Twenty-five endoscopic operations were combined with septoplasty and 92 comprised endoDCR alone. The study was approved by the Sheba Medical Center Institutional Review Board, and it was conducted in accordance with the tenets of the Declaration of Helsinki.

The patients underwent a preoperative comprehensive ophthalmic examination together with irrigation of the nasolacrimal drainage system. They also underwent nasal endoscopy with a $2.6 \mathrm{~mm} 0^{\circ}$ Hopkins rod lens rigid scope after local anesthesia with $10 \%$ xylocaine spray and no mucosal decongestants. Patients were referred to a combined operation of endoDCR + septoplasty if the nasal septum obscured the direct line of view of the most anterior attachment point of the middle nasal turbinate (the middle turbinate axilla) on the side of the planned endoDCR. The other patients were referred to endoDCR alone. Patients with a history of previous lacrimal drainage surgery, lacrimal drainage system malignancy, post-traumatic nasal bony deformity, or canalicular stenosis were excluded.

Postoperatively, the patients were examined at 1 week, 2 months, and 6 months following the surgery. Any inserted silicone stent was removed 2-3 months postoperatively. Surgical success was defined as marked improvement in tearing, as well as by a patent lacrimal pathway irrigation. Patients who complained of persistent epiphora underwent endoscopy of the nasal cavity. Functional success was defined as resolution of epiphora, and anatomical success was defined by patent syringing and by endoscopic evidence of a patent rhinostomy.

Data retrieved from the patients' medical records included demographic characteristics, ocular and lacrimal past history, details of the index operation, complications, and outcomes. The main outcome measures were functional and anatomical success rates. The secondary outcome measure was the rate of postoperative complications.

\section{Surgical technique}

The operations were performed by one senior oculoplastic surgeon (GJBS or AP) and one senior sinus surgeon (AY or JBS). Nasal packing soaked with $3 \mathrm{ml}$ amethocaine $2 \%$ and $1 \mathrm{mg}$ of adrenaline was followed by injection to the lateral nasal wall anterior to the middle turbinate attachment with $1 \%$ lidocaine and $0.1 \mathrm{mg}$ of adrenaline. A $0^{\circ}$ or $30^{\circ} 4 \mathrm{~mm}$ endoscope was used according to the preference of the surgeon. A light source was inserted through the lower punctum and canaliculus until reaching a hard stop, in order to facilitate visualizing the exact position of the lacrimal sac endoscopically. The junction between the frontal process of the maxilla and the lacrimal bone was exposed by elevating a posteriorly based mucosal flap or by removing the mucosa. A $2 \mathrm{~mm}$ rongeur and a $3.2 \mathrm{~mm} 15^{\circ}$ curved diamond burr (Medtronic ENT Xomed, FL, USA) were used to remove the bone overlying the lacrimal sac until the fundus of the sac was exposed. A Bowman's probe (Visitec, Warwickshire, UK) was then inserted to the inferior punctum until tenting of the medial sac wall. The medial wall of the sac was incised vertically with a sickle blade and the entire medial face of the lacrimal sac was resected. A bicanalicular silicone stent was inserted (Crawford, FCI Ophthalmics, Pembroke, MA, USA) and tied over a silicone sleeve.

In cases of significant ipsilateral septal deviation, endoscopic septoplasty was performed prior to the DCR through an incision on the side contralateral to the DNS. After elevation of a subperichondrial flap, septal cartilage and bone were removed as required for complete exposure of the lacrimal area, and the remodeled cartilage was repositioned. The incision was then sutured with 4.0 Vicryl Rapide suture (Ethicon, Johnson \& Johnson, Somerville, NJ, USA). Preseptoplasty and postseptoplasty endoscopic views of nasal cavity of a patient are presented in Fig. 1.

\section{Statistical analysis}

An independent samples $t$-test was used to calculate differences in parametric variables between the two groups (endoDCR and endoDCR + septoplasty). A Chi-square analysis was used to calculate proportional differences between the two groups. The data were analyzed using nonparametric analysis tests for a group with fewer than 30 patients. The overall significance level was set to an alpha of 0.05 (twosided). Statistical analysis was carried out using Microsoft Excel 16.1.1 (Microsoft Corporation, Redmond, WA, USA) and SPSS software version 23.0 (SPSS, Inc., Chicago, IL, USA). Data were shown as mean \pm SD (standard deviation). 
Fig. 1 Endoscopic views of the right nasal cavity of a patient with deviation of the nasal septum and nasolacrimal duct obstruction. a Pre septoplasty: the middle turbinate (arrow head) and the future rhinostomy are in close proximity to the nasal septum (arrow). b Post septoplasty: the future rhinostomy would be sufficiently distant from the nasal septum

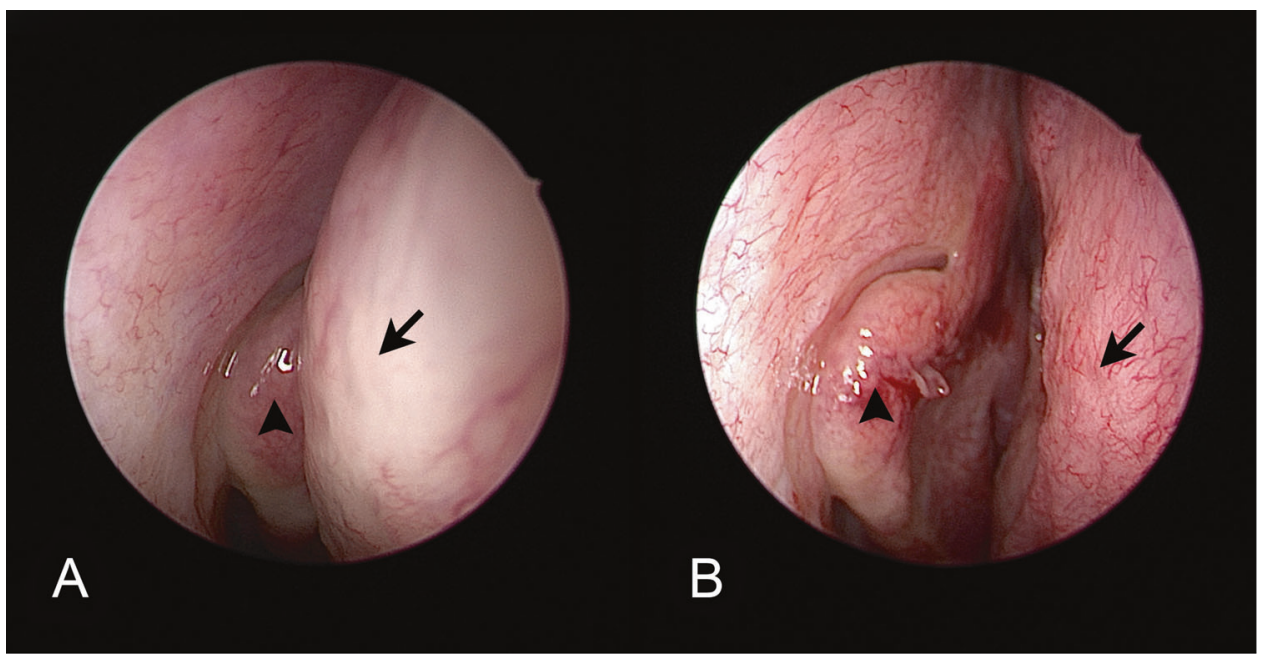

\section{Results}

One hundred and seven consecutive patients who underwent 117 endoDCRs were included in the study $(80.4 \%$ females, $19.6 \%$ males; mean age \pm SD $51.1 \pm 19.5$ years). Ninety-two procedures $(78.6 \%)$ were endoDCR alone (the endoDCR group), and 25 procedures (21.4\%) were endoDCR combined with septoplasty (the endoDCR + septoplasty group). Demographic characteristics, medical history, and lacrimal history of the study population are summarized in Table 1 . Four patients had previously undergone septoplasty for DNS, all due to chronic nasal obstruction, and none of them required revision septoplasty concomitantly with endoDCR in this series. There was no group difference in hypertension, diabetes mellitus, and chronic use of anticoagulants or antiplatelet drugs (patients were instructed to abstain from using these medications for 1 week prior to surgery) (Table 1). There was also no group difference in past lacrimal features such as the frequency of epiphora, punctal discharge, or episodes of dacryocystitis. The groups were also similar in the use of lacrimal imaging as part of the preoperative evaluation process: specifically, five patients in the endoDCR group and one patient in the endoDCR + septoplasty group underwent computed tomography screening and two patients in the endoDCR group underwent dacryoscintigraphy (Table 1).

Both the anatomical and functional success rates were similar for the two groups (Table 2). Postoperative complications included one case of early mild postoperative nasal hemorrhage in the endoDCR + septoplasty group which resolved with nasal packing. The most common lateonset complication was scarring of the rhinostomy (six cases in the endoDCR group and two cases in the endoDCR + septoplasty group), followed by two cases of postoperative nasal mucosal adhesions (one case in each group), two cases of sump syndrome in the endoDCR group, one case of unroofing of the canaliculus in the endoDCR group, and one case of punctal stenosis in the
Table 1 Comparison of demographic features, medical history, and lacrimal preoperative history of 107 patients who underwent 117 endoscopic dacryocystorhinostomies with or without septoplasties

\begin{tabular}{lccc}
\hline & $\begin{array}{l}\text { endoDCR with } \\
\text { septoplasty }\end{array}$ & $\begin{array}{l}\text { endoDCR without } \\
\text { septoplasty }\end{array}$ & $P$ \\
\hline $\begin{array}{l}\text { Age at surgery (years) } \\
\text { (mean } \pm \text { SD) }\end{array}$ & $47.4 \pm 18.1$ & $52.0 \pm 19.7$ & 0.33 \\
Gender, $n$ (\%) & $5(23.8)$ & $16(18.6)$ & \\
$\quad$ Male & $16(76.2)$ & $70(81.4)$ & 0.59 \\
Female & & & \\
Eye, $n$ (\%) & $14(56.0)$ & $35(38.0)$ & \\
Right & $11(44.0)$ & $57(62.0)$ & 0.11 \\
Left & $4(16)$ & $6(6.5)$ & 0.13 \\
Both & & & \\
Medical history, $n(\%)$ & $4(19.0)$ & $21(25.0)$ & 0.56 \\
$\quad$ Hypertension & $4(19.0)$ & $12(14.5)$ & 0.6 \\
Diabetes mellitus & $2(10)$ & $10(12)$ & 0.81 \\
Anticoagulants/ & & & \\
antiplatelets use & & & \\
Lacrimal history, $n(\%)$ & & $77(84.6)$ & 0.34 \\
Epiphora & $23(92.0)$ & $38(41.3)$ & 0.47 \\
Dacryocystitis & $8(33.3)$ & $30(33.0)$ & 0.97 \\
Punctal discharge & $8(33.3)$ & $6(6.5)$ & 0.78 \\
Lacrimal drainage & $1(4.0)$ & & \\
system imaging & & 50 standard & \\
\hline
\end{tabular}

endoDCR endoscopic dacryocystorhinostomy, $S D$ standard deviation

endoDCR + septoplasty group. There were no complications attributed to the septoplasty procedure itself.

\section{Discussion}

DNS is a common nasal finding, with up to $75-80 \%$ of the US population having some degree of DNS [8, 9]. Septoplasty is the third most common head and neck surgery in 
Table 2 Comparison of intraoperative factors and success rates in patients who underwent endoscopic dcryocystorhinostomies with or without septoplasties

\begin{tabular}{llll}
\hline & endoDCR with septoplasty & endoDCR without septoplasty & $P$ \\
\hline Bicanalicular stent inserted, $n(\%)$ & $25(100)$ & $87(94.6)$ & 0.23 \\
Mean duration of stent, weeks \pm SD & $9.6 \pm 6.7$ & $10.1 \pm 6.3$ & 0.76 \\
Mucosal flap elevated, $n(\%)$ & $17(68.0)$ & $44(49.4)$ & 0.1 \\
Anatomical success, $n(\%)$ & $22(88.0)$ & $82(90.1)$ & 0.76 \\
Functional success, $n(\%)$ & $19(76.0)$ & $79(86.8)$ & 0.18 \\
\hline
\end{tabular}

endoDCR endoscopic dacryocystorhinostomy, $S D$ standard deviation the US, with over 250,000 cases performed annually [10]. The indication for septoplasty is usually chronic nasal airway obstruction. Interestingly DNS is more common among patients with primary acquired NLDO (60\% vs. $36 \%$ in a control group [11]), and it corresponds in laterality to the side of the obstruction in about $90 \%$ of the cases [11, 12]. The reported rate of concomitant endoDCR and septoplasty for DNS is considerable, and ranges from 11.9 to $57 \%$. $[2,3,7,13-16]$ In our series, the rate of concomitant endoDCR and septoplasty was $21.4 \%$ of patients who presented with NLDO.

Our data showed that integrating an additional procedure (septoplasty) that was not performed for its original indication but rather for facilitating the main surgical intervention (endoDCR) yields results that are comparable with those for cases of endoDCR without septoplasty in terms of surgical success and complications. To the best of our knowledge, this is the largest series that compares the contribution of adjunctive septoplasty to the success of endoDCR compared with endoDCR alone. Figueira et al. [2] reported a large series of 576 patients who underwent endoDCR, of whom 81 had concomitant endonasal procedures (septoplasty, turbinectomy, and polypectomy): the results were similar to those for the nonconcomitant procedures, but there was no reference to septoplasty alone. Fayet et al. [3] demonstrated that higher rates of anterior middle turbinectomies and septoplasties performed during endonasal DCRs yielded higher rates of elimination of epiphora, but, again, septoplasty was not evaluated in isolation and it comprised only one quarter of all the endonasal procedures in that series.

Chan et al. reported a $90 \%$ success rate of combined external DCR with septoplasty in a group of eight patients [17], but with no comparison with a control group. Other studies described frequencies of concomitant endonasal procedures during endonasal DCR, but none compared the impact on the success rate of a combined surgery with that of DCR alone. [7, 14-16] Among these studies, Bayraktar et al. had the highest rate of endoDCR with concomitant septoplasty $(57.1 \%)$ in their bilateral NLDO group, while the rate of concomitant septoplasty in the unilateral NLDO group was much lower (16.7\%) [16]. Revisions with concomitant septoplasty have been described in the literature, but the surgical success rates were never compared with those for DCR alone [6, 17-21]. We believe that our finding that the outcomes of the combined approach were equivalent to those of the DCR alone is highly significant, since an untreated DNS may cause failure of an otherwise successful DCR due to the proximity of the septum to the healing mucosal tissues, resulting in adhesions. Furthermore, septoplasty is often performed when septal deviation impedes surgical access during an endoDCR, regardless of airway obstruction symptoms [22].

Choosing an external DCR approach may help in avoiding the difficulty in access to the lacrimal sac fossa, but the untreated DNS will still influence the outcome of the operation. Severe DNS that was responsible for surgical failure was significantly more frequent postoperatively in cases of a previously failed external DCR than in cases of a previously failed endoscopic DCR (23\% vs. 3.5\%) [6]. Furthermore, failed primary external DCRs revised with an endoscopic approach have been shown to require higher rates of septoplasty (16\%) compared with revised endoscopic DCRs (only $1.7 \%$ needed additional septoplasty) [6].

EndoDCR has been suggested as being advantageous in revision cases due to the ability to address the cause of initial failure at the time of revision surgery [5,23]. Treating a DNS and other endonasal anomalies at the time of the initial surgery in order to minimize the need for revision surgery has been advocated in several pubications [13, 17].

We did not encounter any septal perforations, external nose deformations, or other complications related to septoplasty in our current study, in line with other reports $[2,7]$. This can be attributed to the use of a conservative technique of limited upper septoplasty aimed primarily to improve endoscopic access to the lacrimal sac. In our opinion, combined endoDCR and septoplasty requires the collaboration of an ear, nose, and throat surgeon together with the oculoplastic surgeon. We believe it is advantageous to combine the knowledge and experience of both disciplines, when feasible.

The strength of the current study is the specific comparison of endoDCR alone to endoDCR + septoplasty without other concomitant endonasal procedures. The major limitation of the study is its retrospective design; therefore, further randomized control trials are needed to confirm the results. 
In summary, our findings support the performance of a septoplasty in every case of endoDCR when access to the lacrimal sac is not optimal due to a DNS and technical difficulties are anticipated. Simultaneous septoplasty does not expose the patients to further complications, and the surgical success rate is similar to that of endoDCR alone.

\section{Summary}

\section{What was known before}

The ability to perform a concomitant endoscopic septoplasty for correction of a DNS is an advantage of endoDCR compared to external DCR.

Septoplasty is sometimes performed to improve the execution and the outcome of the endoscopic DCR

\section{What this study adds}

Integrating a septoplasty, that was not performed for its original indication but rather for facilitating the main surgical intervention (endoDCR), yields results that are comparable to those for cases of endoDCR without septoplasty in terms of surgical success and complications

\section{Compliance with ethical standards}

Conflict of interest The authors declare that they have no conflict of interest.

Publisher's note Springer Nature remains neutral with regard to jurisdictional claims in published maps and institutional affiliations.

\section{References}

1. Marcet MM, Kuk AK, Phelps PO. Evidence-based review of surgical practices in endoscopic endonasal dacryocystorhinostomy for primary acquired nasolacrimal duct obstruction and other new indications. Curr Opin Ophthalmol. 2014;25:443-8.

2. Figueira E, Al Abbadi Z, Malhotra R, Wilcsek G, Selva D. Frequency of simultaneous nasal procedures in endoscopic dacryocystorhinostomy. Ophthalmic Plast Reconstr Surg. 2014;30:40-3.

3. Fayet B, Katowitz WR, Racy E, Ruban JM, Katowitz JA. Endoscopic dacryocystorhinostomy: the keys to surgical success. Ophthalmic Plast Reconstr Surg. 2014;30:69-71.

4. Elmorsy SM, Fayk HM. Nasal endoscopic assessment of failure after external dacryocystorhinostomy. Orbit. 2010;29:197-201.
5. Demarco R, Strose A, Araújo M, Valera FC, Moribe I, AnselmoLima WT. Endoscopic revision of external dacryocystorhinostomy. Otolaryngol Head Neck Surg. 2007;137:497-9.

6. Paik JS, Cho WK, Yang SW. Comparison of endoscopic revision for failed primary external versus endoscopic dacryocystorhinostomy. Clin Exp Ophthalmol. 2013;41:116-21.

7. Ali MJ, Psaltis AJ, Wormald PJ. The frequency of concomitant adjunctive nasal procedures in powered endoscopic dacryocystorhinostomy. Orbit. 2015;34:142-5.

8. Aaronson NL, Vining EM. Correction of the deviated septum: from ancient Egypt to the endoscopic era. Int Forum Allergy Rhinol. 2014;4:931-6.

9. Andrades P, Cuevas P, Danilla S, Bernales J, Longton C, Borel C, et al. The accuracy of different methods for diagnosing septal deviation in patients undergoing septorhinoplasty: a prospective study. J Plast Reconstr Aesthet Surg. 2016;69:848-55.

10. Bhattacharyya N. Ambulatory sinus and nasal surgery in the United States: demographics and perioperative outcomes. Laryngoscope. 2010;120:635-8.

11. Singh S, Alam MS, Ali MJ, Naik MN. Endoscopic intranasal findings in unilateral primary acquired nasolacrimal duct obstruction. Saudi J Ophthalmol. 2017;31:128-30.

12. Taban M, Jarullazada I, Mancini R, Hwang C, Goldberg RA. Facial asymmetry and nasal septal deviation in acquired nasolacrimal duct obstruction. Orbit. 2011;30:226-9.

13. Ali MJ, Psaltis AJ, Bassiouni A, Wormald PJ. Long-term outcomes in primary powered endoscopic dacryocystorhinostomy. $\mathrm{Br}$ J Ophthalmol. 2014;98:1678-80.

14. Nussbaumer M, Schreiber S, Yung MW. Concomitant nasal procedures in endoscopic dacryocystorhinostomy. J Laryngol Otol. 2004;118:267-9.

15. Tsirbas A, Wormald PJ. Mechanical endonasal dacryocystorhinostomy with mucosal flaps. Br J Ophthalmol. 2003;87:43-7.

16. Bayraktar C, Simsek A. Increased concomitant nasal procedure frequency in bilateral endoscopic dacryocystorhinostomy. J Craniofac Surg. 2017;28:980-2.

17. Chan DM, Golubev I, Shipchandler TZ, Nunery WR, Lee HB. Improving outcomes by combining septoplasty with primary external dacryocystorhinostomy. Am J Otolaryngol. 2014;35: 309-12.

18. Ari S, Kürşat CA, Sahin A, Gün R, Kiniş V, Caça I. Outcomes of revision external dacryocystorhinostomy and nasal intubation by bicanalicular silicone tubing under endonasal endoscopic guidance. Int J Ophthalmol. 2012;5:238-41.

19. Liang J, Hur K, Merbs SL, Lane AP. Surgical and anatomic considerations in endoscopic revision of failed external dacryocystorhinostomy. Otolaryngol Head Neck Surg. 2014;150:901-5.

20. Yarmohammadi ME, Ghasemi H, Jafari F, Izadi P, Nadoushan MJ, Chin NS. Teamwork endoscopic endonasal surgery in failed external dacryocystorhinostomy. J Ophthalmic Vis Res. 2016; $11: 282-6$

21. El-Guindy A, Dorgham A, Ghoraba M. Endoscopic revision surgery for recurrent epiphora occurring after external dacryocystorhinostomy. Ann Otol Rhinol Laryngol. 2000;109:425-30.

22. Knisely A, Harvey R, Sacks R. Long-term outcomes in endoscopic dacryocystorhinostomy. Curr Opin Otolaryngol Head Neck Surg. 2015;23:53-8.

23. Korkut AY, Teker AM, Yazici MZ, Kahya V, Gedikli O, Kayhan FT. Surgical outcomes of primary and revision endoscopic dacryocystorhinostomy. J Craniofac Surg. 2010;21:1706-8. 